is a very rare occurrence. No such result has occurred in my practice, though two cases have come under my care in the infirmary - one not long after the suppuration had commenced, and the other only after the globes had become destroyed.

The first case was that of a young woman, unmarried, aged twenty-three, and she was admitted into the Fir Vale Workhouse on Nov. 14th, 1882, where our present housesurgeon, Mr. C. F. Coombe, saw her, whilst acting as resident medical officer to that institution, and he has kindly supplied most of the particulars which follow. At this time ghe presented well-marked enlargement of both lobes of the thyroid gland, with great prominence of the eyeballs. She had frequent attacks of very severe palpitation, and systolic bruits were heard both at the base and apex of the heart. During the next fortnight the exophthalmos increased considerably, and became so great that she could not cover the globes with the eyelids. Ulcers appeared on the cornea, gpreading and assuming a sloughing character. On Dec.2nd she was admitted into the Sheffield General Infirmary, under Dr. Bartolome, and on the 4th was transferred to me. Shortly after admission she was attacked with erysipelas (facial), and died on Dec. 12th. No post-mortem examination was allowed. When she came under my observation both corneæ were extensively sloughed, altogether past recovery. No cause was assigned for the disease, but her condition from the first when under my care was such as to preclude the possibility of taking detailed particulars of her case.

The second instance was in a married woman aged forty. She was admitted into the Sheffield General Infirmary in May last (1886) with both eyeballs destroyed by sloughing of the corneæ. The thyroid gland was markedly enlarged; there was palpitation and a loud hæmic murmur (basic). There was the excitable nervous disposition met with in patients having Graves' disease, and "flushings " were likewise experienced. The eyelid symptoms were obscured as a consequence of the sloughing of the eyeballs, but Stellwag's symptom (retraction of eyelids) was undoubtedly present.

A remark occurs to me respecting the condition of the thyroid gland in these cases of exophthalmic goitre. Frequently one lobe is larger than the other, and in recorded cases in which the side is particularised, the right is the one referred to. Anatomists, however, state that the right lobe is normally larger than the left. Have clinicians borne in mind sufficiently the anatomical fact?

Monocular cases.-Generally speaking, Graves' disease is bilateral, though not unfrequently it is more marked on one side of the body than the other. Several cases are on record in which the eye symptoms have been confined to one eye. Dr. Hill Griffiths stated at the Ophthalmological Society that the eye symptoms in his thirty cases were bilateral in twenty-three instances; in four the right only was affected, and the left in three. In addition to the patient already referred to (No. 1), I have notes of two other cases.

The first occurred in a female, aged fifty-four, who consulted me in 1877. She suffered greatly from palpitation; pulse 150. Exophthalmos (right side); lids closed over the eyeball but not easily. Had only been married recently; her health was poor, and she had had a great deal of nursing slept badly. Menstruation ceased a year ago. The thyroid gland was enlarged; my note does not say whether equally 80 , or whether one side was larger than the other. 1 only saw her a few times.

The next case was a domestic servant aged twenty-three, Tho was first seen in June, 1884. For three or four months the left eye was noticed to be prominent, and had been much worse during the last two months. The retraction of the eyelid (Stellwag's symptom) was well marked, and had been so for two months, the patient stating that the eye had been as wide open during that period as it was at the present time. The eye was prominent, and Graefe's sign loss of coördinated movements in descent of eyeball and upper eyelid, was present. The eye was myopic; $\vec{V}=$ fingers, and was but little improved by any - glass. There was divergent squint; there were extensive choroidal changes in the region of the macula. She had been aware of the badness of vision with the eye for many years. The right eye was hypermetropic, and the symptoms present in the left were absent. She suffered a great deal from palpitation; there was a hæmic cardiac bruit, audible with first sound at base and into the neck. Goitre was present, and had only been noticed two or three weeks-not large but distinct, and, if anything, the right was the larger side. No shock or other apparent cause. Menstruation was frequent.
She was introduced at one of our meetings some time after this. She remained under my care, and in the following November it seemed as if the right eye would also become affected; but it never did so. At the end of twelve months the following note was made: "She has been at Scarborough, and is in much better health. The prominence of the left eye is now very much less, but she thinks it is not quite as it was before the attack commenced. The right eye is quite good. No flushings now, nor palpitations. Has lost her nervousness. Catamenia regular."

A theory to be satisfactory must be able to account for the whole train of symptoms. The one that seems to fall in best with this condition is the central lesion theory brought forward by Sattler, and which has found in these islands a very able advocate in Dr. W. A. Fitzgerald of Dublin. ${ }^{2}$ Briefly, Sattler assumes a lesion of the vaso-motor centre that presides over the vaso-motor nerves of the thyroid gland and orbital tissues, and it is inferred from the trequent association of goitre and exophthalmos that these centres must be close together. The cardiac syuptoms he ascribes to lesion of the cardio-inhibitory centre for the vagus, and Graefe's symptom is explained on the assumption that there is a centre governing the associated downward movements of the eye and eyelids, just as the associated movements of both eyes. He coincides in Stell wag's opinion that the widening of the palpebral opening is due to a lesion of the reflex centres, which are set in motion by stimuli from the retina, cornea, and conjunctiva. The experiments of Filehne on the restiform bodies have given considerable support to this theory, these being the parts through which the nerve paths from the vaso-motor centres and vagal centres pass. In one case he succeeded in inducing the three cardinal symptoms, and in the others a varying association of the symptoms. Moreover, the interesting association of diabetes in some cases of Graves' disease is well known, and it is worthy of notice that the region described as the diabetic puncture is close to the vaso-motor area. Do not the cases referred to in this paper lend support to such a central theory? In other diseases we are accustomed to regard a lesion as spreading and gradually encroaching on contiguous areas, either by direct extension or by irritation of surrounding tissues. May we not, therefore, regard the cases in which the lid symptoms are among the earliest as instances in which the lesion has only affected the centres governing the coördinated downward movement of the globe and eyelid, with the lid reflexes generally closely associated, the other centres becoming implicated later on, with a consequent further development of symptoms? Or are there cases which stop at a lesion of the lid centres?

COLOTOMY FOR MALIGNANT DISEASE OF THE RECTUM, WITH NEARLY PERFECT CONTROL OVER THE ARTIFICAL ANUS A YEAR AFTER THE OPERATION.

\author{
Br HARRISON CRIPPS, F.R.C.S., \\ ASSISTANT-SURGEON TO ST. BARTHOLOMEW'S HOSPITAL.
}

Tres patient, a woman aged fifty, was kindly placed under my care at the hospital by Dr. Griffith. I am indebted for the following notes to Mr. Balgarnie, her dresser.

The woman was admitted on Dec. 15th, 1885. She dates her present illness from the spring, when she first noticed pain in the lower part of the back, and soon afterwards pain during defecation. The character of her motions gradually altered, becoming smaller in size, and at times flattened and generally tinged with blosd. The frequency of her stools increased, at first to three or four times a day, but latterly much more often. She has been getting thinner, and at times has much severe pain, which she described as like "labour pains."

The patient on admission looked weak and anæmic, with a feeble pulse. She had nearly constant pain, and was much distressed by a troublesome diarrhcea, having to go to stool as often as ten or twelve times in the night. The act of defecation was very painful, and nearly always attended by loss of blood. On examination the anus appeared healthy, and on 
ntroducing the finger the mucous membrane for about three inches felt smooth and natural. The finger then came in contact with a mass feeling not unlike an enlarged cervix uteri. Around this was a cul-de-sac, deeper posteriorly than anteriorly. The mass itself was hard and nodular. The opening through it would only admit the tip of the finger, and the bowel at the constricted point was firmly fixed to the surrounding structuree.

The patient, not wishing then to stay in the hospital, left, but was readmitted on Feb. 1st. She had become weaker, and her symptoms had increased since her discharge. The mass also had grown considerably, and could now be felt to be within an inch and a half of the anus. On Feb. 8th, 1886, the case being considered unsuitable for excision, lumbar colotomy was performed by Mr. Harrison Cripps. The various layers between the skin and the lumbar fascia were purposely not divided on quite the same level, and the opening in the fascia was considerably above that in the skin, so that the bowel when drawn out ran obliquely for a short distance between the skin and the fascia. T'he wound united by first intention. No motion passed through it for a week. During the next six weeks a certain amount of fæces passed through the restum as well as through the wound. This gradually ceased, and when she was discharged in March everything passed through the artificial anus. She improved very much whilst in the hospital, and was comparatively free from pain.

April, 1887.-The patient is not only alive, but wonderfully improved; and notwithstanding that the local growth bas made some advance, her general health and strength is far better than it was a year ago. She is no longer troubled with diarrhoea, but has one good motion daily through the artificial anus, and she has been able to attend as usual during the year to her domestic duties. The artificial anus readily admits the forefinger, and the mucous membrane is exactly on the level of the skin. There is no sign of cicatricial tissue round the orifice, which is perfectly soft and dilatable. The patient knows when she is going to pass a motion, which she has the power of controlling, there being no involuntary escape. In fact, the patient herself says she is no more troubled with the artificial anus than when the opening was in the natural situation. The patient was shown at the Medical Society.

After carefully watching a large number of cases of rectal cancer, in some of which colotomy had been performed, while in others nature had been allowed to take its course, I do not hesitate tospronounce strongly in favour of the operation. The relief to the symptoms is very marked, the constant diarrhoe and tenesmus which forms so frequent and distressing a complication being entirely relieved. The downward course of the patient is certainly made far easier, and the miserable termination of life by obstruction or perforation prevented. Moreover, I feel confident that the progress of the growth is retarded in consequence of the bowel acquiring physiological rest by the cessation of its function. That the rapidity of malignant growth depends much on the activity and consequent blood-supply of the organ affected admits of no doubt, and as an example may be instanced the relative rapidity with which cancer grows in the breast of a pregnant or suckling woman. So that, apart from life being indirectly prolonged by the relief which colotomy affords to distressing symptoms, I feel confident that the growth itself is often retarded, and rendered comparatively quiescent when the rectum is placed at rest by the operation. Colotomy is in no sense a rival operation with that of excision. Excision is doubtless the proper operation when the disease is within easy reach and still confined to the walls of the rectum, thus admitting of a free and complete removal. Such favourable conditions are, however, unfortunately exceptional, the disease either having commenced too high or spread too far by the time the case has come under observation to admit of local interference. The practical question that arises is as to what period in the course of a case of rectal cancer colotomy should be performed. In answering this question, I am in complete accord with Mr. Reeves, who advocates the performance of the operation so soon as symptoms of stricture become prominent. The troublesome diarrhoea, pain, tenesmus, and blood-stained discharge owe their origin far more to the retention of scybala above the contracted part than to the actual disease itself. To wait until the unfortunate sufferer is at death's door, worn out by the constant discharge, or by the actual occurrence of complete obstruction, is not only to have deprived the patient of the benefit of the operation, but to perform it at a time when it becomes a dangerous procedure. The operation, if performed before the patient is much reduced in strength, has but little risk; indeed, I can say in my own practice that I have hitherto had no death as the immediate result of the operation when performed for malignant disease. It is essential, if the patient is to derive full benefit from the operation, that care should be tcken to prevent the opening from contracting. It is of great im. portance, with this view, to get union of the bowel with the skin by first intention. If this occurs there is little or no tendency to contract. On the other hand, if the bowel falls away from the skin the granulating tiseue with which the interval is occupied is nearly sure to contract on healing, In one case in which 1 recently operated half the benefit was lost from this cause, for, on seeing the patient two months after the operation, the opening would barely admit the finger. If there is any tendency to contraction, it is best prevented by allowing the wound to heal over a full-sized plug. I regard it as a great advantage to make the opening through the abdominal walls as valvular as possible, and this is best accomplished by making the incision through the skin considerably lower than that through the faccia If the opening is made in this way, and care taken during the healing process to prevent contraction, the patient will have little or no difficulty in managing the artificial anus. In the case recorded, the part was perfectly clean, the patient had one motion daily, and had control over the bowel, while she was able to do her daily work without annoyance of any kind.

Stratford-place, Oxford-street, $W$.

\section{EXTIRPATION OF RUPTURED UTERUS.}

By E. C. ANDREWS, B.A., M.B., B.C. CANTAB., S., sCHOLAR oF ST. JOHN'S COLLEGE, UAMTBRIDGE, AND SEYIOR ODSTETRIC RESIDENT AT GUY'S HOSPITAL.

ON September 12th, at 5.30 P.M., one of the extern midwifery attendants was called in to attend the fifth labour of Mrs. S - - who was said to have had pains for twenty-four hours. She was a delicate woman of short stature and her previous confinements had all been normal. Vaginal examination showed the os fairly dilated, membranes unruptured, and the vertex presenting resting on the pubes. The pains were of medium frequency, and apparently those of the first stage of labour. At 6.30 P.M., the pulse being then about 120 , the membranes ruptured, and the pains increased in force and frequency, culminating about 8 P.M. in exceedingly strong expulsive efforts, which affected the progress of the head but little, if at all. Immediately after these the patient complained of intense pain in the stomach, there was marked collapse, and all labour pains ceased.

I was now summoned to the case, and found the patient nearly pulseless, pale, and exhausted, but quite conscious, and the head on the brim of the pelvis and rather movable. Recognising the urgent necessity for immediate delivery, I directed the abdomen to be well supported by the extern, introduced the long forceps, and proceeded to deliver the child. No difficulty was experienced in the introduction of the blades, and but very gentle traction was required. Fearing that the perineum might rupture, I withdrew the forceps as soon as the head was well down upon it, but instead of expulsion taking place, the head receded into the vagina. I again applied the forceps, and delivered without further difficulty. The child was a large one, weighing $9 \frac{1}{2} \mathrm{lb}$, was stillborn, and the delivery was followed by a gush of blood, though the uterus appeared firmly contracted. After waiting a few minutes, finding that the placenta did not come away, I introduced my hand and found that it passed through a rent in the uterine wall into the abdominal cavity. Following up the cord with my fingers, I discoverd the placenta in the abdominal cavity above the uterus, and removed it without difficulty. An injection of two-thirds of a grain of ergotine was now administered. The pulse was quite imperceptible and the patient apparently dying. An hour later the patient had rallied considerably; the pulse was 140, of fair force, and there had been no furtber hæmorrhage from the vagina. About 10.30 P.M., however, the pulse was much feebler, and coils of intestine had come down through the rent into the vagina. 